\title{
N75 to P100 Peak to Peak Amplitude
}

National Cancer Institute

\section{Source}

National Cancer Institute. N75 to P100 Peak to Peak Amplitude. NCI Thesaurus. Code C117887.

The magnitude, or height, of the P100 wave of the visual evoked potential assessment waveform. This assessment calculates the height from the N75 negative peak to the P100 positive peak. 